\title{
New record of Pantophthalmus pictus (Wiedemann, 1821) (Diptera, Pantophthalmidae) in the Cerrado vegetation of central Brazil
}

\author{
José Roberto Pujol-Luz ${ }^{1,2}$ \& Giovanna Souto Morgado ${ }^{1,3}$ \\ 1 Universidade de Brasília (UNB), Instituto de Ciências Biológicas (IB), Departamento de Zoologia. Brasília, DF, Brasil. \\ 2 ORCID: 0000-0002-8621-4856. E-mail: jrpujol@unb.br \\ 3 E-mail: giovannamorgado@hotmail.com
}

\begin{abstract}
Pantophthalmus pictus (Wiedemann, 1821) (Diptera, Pantophthalmidae) has been reported to occur in south and southeastern Brazil, Paraguay and Argentina. Here we report the first occurrence of these rare flies in the Cerrado vegetation of central Brazil. We also provide an updated list of the geographical distribution of the 20 species of the genus Opetiops Enderlein, 1921 (1 species) and Pantophthalmus Thunberg, 1919 (19 species) in the Neotropical region.
\end{abstract}

Key-Words. Biogeography; Brazilian central plateau; Neotropical region; Taxonomy; Timber flies.

\section{INTRODUTION}

The Pantophthalmidae is a small family of 'orthorrhaphous' Brachycera flies found exclusively in the Neotropical region. They are rare flies and little represented in the entomological collections. Currently are recognized 20 valid species in two genera: Opetiops Enderlei, 1921 and Pantophthalmus Thunberg, 1919 (Val, 1976, 1992; Papavero, 2009a). They are very large flies (18-45 mm), mostly dark brownish or reddish with longitudinal stripes on the scutum; some males silver pubescent. The wings are hyaline to infuscated with yellowish or brownish spots, and varying patterns. The eyes are very large, occupying most of the head. The females are dichoptic, and the males holoptic. The face has a usually conical protuberance of varying size and shape.

Papavero (2009b) provided an annotated list of pantophthalmids species (larvae and imagoes) and its host trees. The larvae of Timber flies live in dead or alive trees (Carrera \& d'Andretta, 1957), forming galleries where they feed on accumulated organic matter and/or its fermentation (Andrade, 1930; Greene \& Urich, 1931; Thorpe, 1934). The larvae of Pantophthalmus pictus (Wiedemann, 1821) uses a large number of native (Araucariaceae, Bombacaceae, Fabaceae, Lauraceae, Moraceae, Rhamnaceae, Rutaceae, Sapotaceae) and introduced trees (Aceraceae, Arecaceae, Casuarinaceae, Fabaceae, Fagaceae, Juglandaceae, Magnoliaceae, Moraceae, Myrtaceae, Platanaceae, Rosaceae, Salicaceae) (Papavero, 2009b).

The most accurate geographical distribution of pantophthalmids was produced by Val (1976).
Later, other records were obtained for Colombia (Amat, 2005; Wolff et al., 2016), Brazil (Papavero, 2009a; Fachin et al., 2017), and Argentina (PujolLuz \& Pujol-Luz, 2014) (Table 1). Pantophthalmus pictus (Figs. 1-2) has been reported to southern and southeastern Brazil, Paraguay and Argentina.

The Cerrado is the second major Brazilian Biome and it is considered one of the biodiversity hotspots of the planet (Myers et al., 2000). It occupies approximately $21 \%$ of the national territory, extending through the central Brazilian Plateau, where the Distrito Federal is located (Fig. 3). In this region there are few studies on Diptera (e.g. Biavati et al., 2010; Harterreiten-Souza et al., 2014).

Here we report the first occurrence of $P$. pictus in the core area of the Cerrado vegetation in central Brazil.

\section{MATERIAL AND METHODS}

Specimens were collected in the neighborhood of urban parks in the cities of Brasília and Águas Claras, Distrito Federal. These sites contain both native Cerrado vegetation and introduced trees. P. pictus ( 1 male and 2 females) were housed in the Entomological Collection of the University of Brasília (DZUB) and labeled as: Brasília/DF, Lago Norte [-15.7381; -47.8583], 21/10/2000, R. Abad col., 1 female (UNB-016296); Brasília/DF, Asa Norte, Quadra 415N [-15.7662; -47.8829], 18/10/2004, Joselito col., 1 male (UNB-016273); Águas Claras/ DF [-15.8372; -48.0258], 16/07/2015, K.P.O. Name col., 1 female (UNB-016331). 


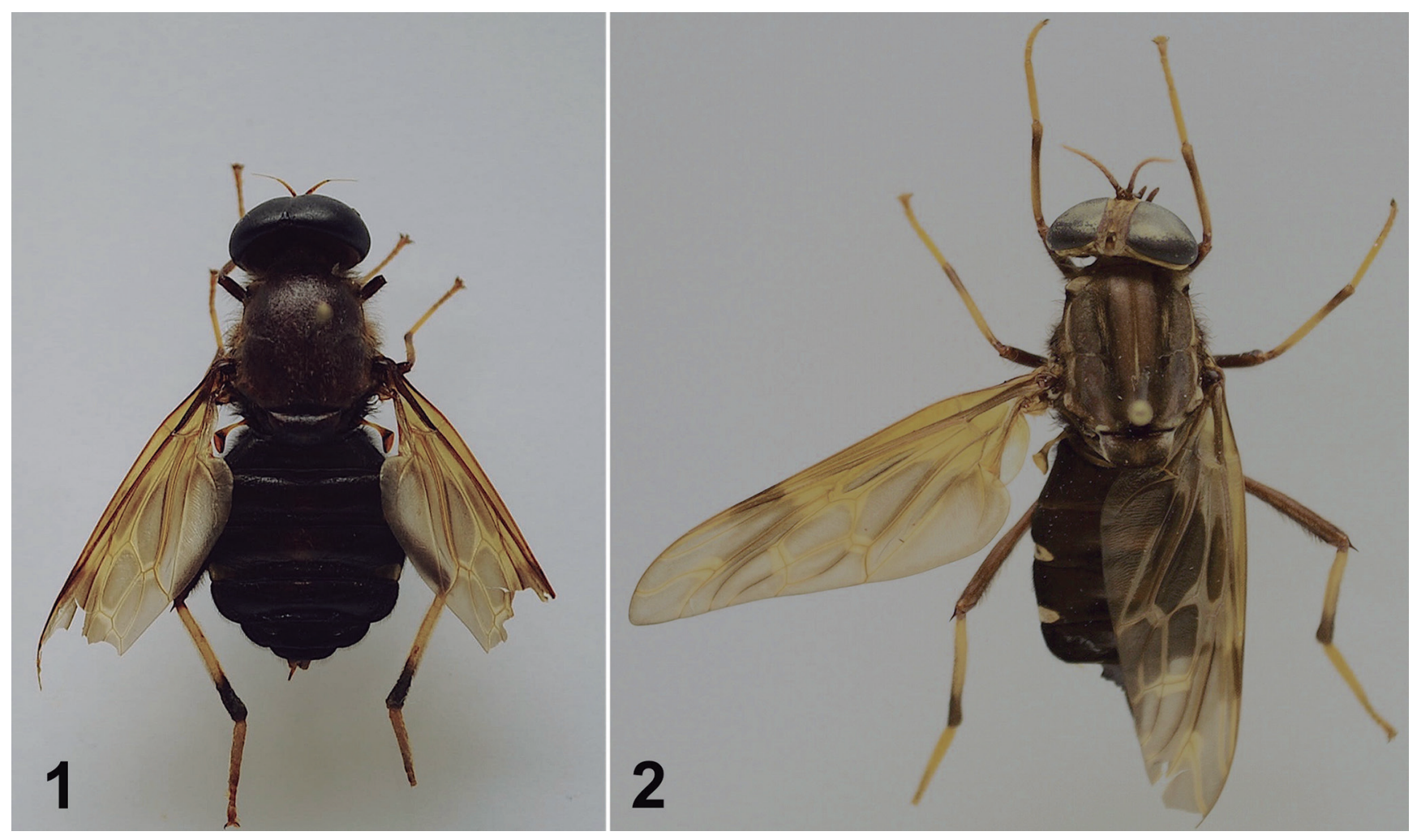

Figures 1-2. Pantophthalmus pictus (Wiedemann). (1) female Male (total length =33 mm). (2) Female (total length = $35 \mathrm{~mm}$ ).

The Generic Mapping Tools (Wessel \& Smith, 1998) was utilized to produce a distribution map. Location records cited by Val (1976) were updated and the approximate coordinates converted for decimal degrees: Argentina. Misiones (1 female) [-27.4269; -55.9967]; Puerto Bemberg, Puerto Libertad (1 male, 1 female) [-25.9210; -54.5839]; San Antonio (1 female) [-26.0572; -53.7365]; San Ignacio (1 male) [-27.2575; -55.5354]. Brazil. Espírito Santo: Sooretama (1 female) [-19.0983; -40.1862]; Minas Gerais: Uberaba (1 male, 1 female) [-19.7473; -47.9391]. Paraná: Matelândia (1 female) [-25.2426; -53.9785], Rolândia (2 males) [-23.3160; -51.3768]. Rio de Janeiro: Rio de Janeiro (2 males, 2 females) [-22.9068; -43.1728], Magé [Barreiras] (1 female) [-22.6566; -43.0398], Nova Friburgo [Mury] (1 female) [-22.3334; -42.4871], Paineiras (2 females) [-22.9475; -43.2095], Petrópolis (1 female) [-22.5112; -43.1779], Rio Grande do Sul: Cerro Largo (1 female) [-28.1437; -54.7192], São Salvador (1 female) [-27.2774; -53.4296]. Santa Catarina: Corupá (2 females) [-26.4355; -49.2452], Itapiranga (4 females) [-27.1698; -53.7108], Mafra (1 female) [-26.1164; -49.8091], Nova Teutônia (1 female) [-27.1612; -52.4167], Rio Natal (1 female) [-26.3659; -49.2897], Rio Vermelho (1 female) [-27.4914;-48.4162]. São Paulo: Cássia dos Coqueiros (1 male) [-21.2818; -47.1687], Cotia [Morro Grande] (1 female) [-23.6026; -46.9914], Limeira ( 1 female) [-22.5838; -47.4097], Piracicaba (3 males, 2 females) $[-22.7342 ;-47.6480]$, Rio Claro $(4$ males, 3 females) [-22.4149; -47.5651]; São Paulo ( 1 male, 5 females) [-23.5505; -46.6333]. Paraguay. Concepción (1 female) [-23.4214; -57.4344], Sapucai (1 female) [-25.6616; -56.9604], Guaira: Villarrica (1 female) [-25.7760; -56.4495]. We also used a Nikon D-700 camera for pictures.

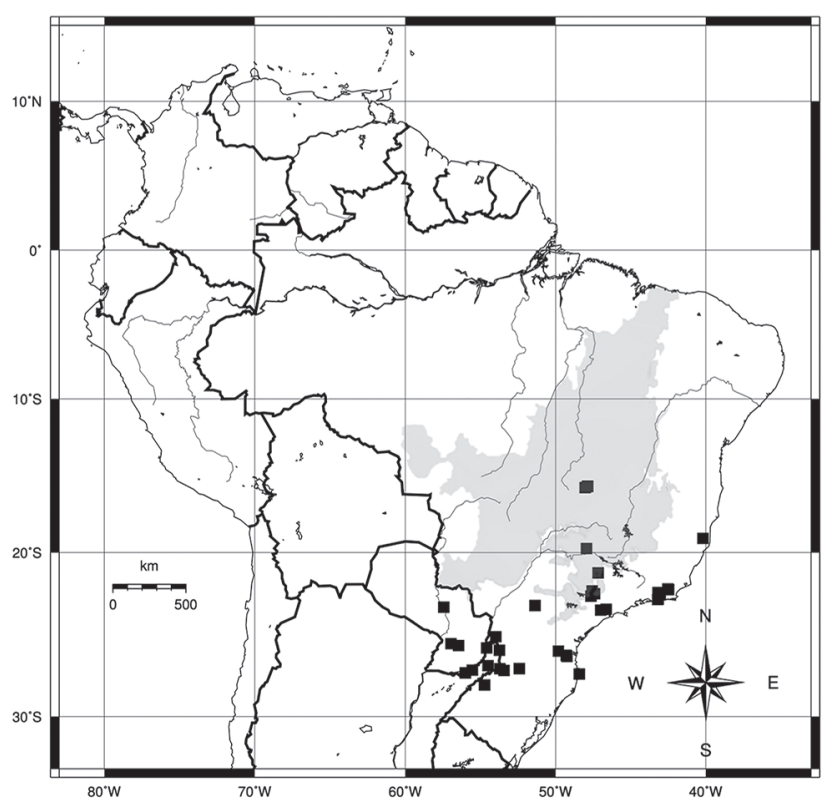

Figure 3. Pantophthalmus pictus (Wiedemann). Records of geographical distribution (Argentina, Brazil and Paraguay). Cerrado Bioma (grey area).

\section{RESULTS}

In spite of the wide distribution of Pantophthalmus in Brazil (Val, 1976; Papavero, 2009a), only two species have been recorded in the Cerrado vegetation: P. planiventris (Wiedemann, 1821) in the Goiás State (city of Anápolis) and P. vittatus (Wiedemann, 1828) in Mato Grosso State (city of Cuiabá). Except by a single 
Table 1. Records of geographical distribution of the genus Opetiops Enderlein and Pantophthalmus Thunberg. (NR $=$ new record).

\begin{tabular}{|c|c|c|}
\hline Timber flies species & Geographic record & Brazil (States) \\
\hline \multicolumn{3}{|l|}{ Opetiops Enderlein } \\
\hline 0. alienus (Hermann) & $\begin{array}{l}\text { Panama, Colombia, Peru, Brazil, } \\
\text { Paraguay }\end{array}$ & Santa Catarina \\
\hline \multicolumn{3}{|l|}{ Pantophthalmus Thunberg } \\
\hline P. argyropastus (Bigot) & Costa Rica & - \\
\hline P. batesi Austen & $\begin{array}{l}\text { Guyana, French Guiana, Peru, } \\
\text { Brazil, Argentina }\end{array}$ & Acre, Amazonas \\
\hline P. bellardii (Bigot) & $\begin{array}{l}\text { Mexico, Guatemala, El Salvador, } \\
\text { Nicaragua, Costa Rica, Panama, } \\
\text { Colombia, Venezuela, Ecuador, } \\
\text { Peru, Bolivia, Brazil }\end{array}$ & - \\
\hline P. chuni (Enderlein) & $\begin{array}{l}\text { French Guiana, Ecuador, Peru, } \\
\text { Brazil, Bolivia, Argentina }\end{array}$ & Amazonas \\
\hline P. comptus Enderlein & $\begin{array}{l}\text { Belize, Guyana, Surinam, French } \\
\text { Guiana, Peru, Brazil }\end{array}$ & Amazonas \\
\hline P. engeli (Enderlein) & Panama & - \\
\hline P. facetus (Enderlein) & Panama & - \\
\hline P. frauenfeldi (Schiner) & $\begin{array}{l}\text { Colombia, Venezuela, Ecuador, } \\
\text { Peru, Bolivia }\end{array}$ & - \\
\hline P. kerteszianus (Enderlein) & $\begin{array}{l}\text { Panama, Colombia, Peru, Bolivia, } \\
\text { Brazil }\end{array}$ & $\begin{array}{l}\text { Amazonas, Pará, Mato Grosso } \\
\text { do Sul }\end{array}$ \\
\hline P. pictus (Wiedemann) & Brazil, Argentina, Paraguay & $\begin{array}{l}\text { Distrito Federal (NR), Minas } \\
\text { Gerais, Espírito Santo, Rio de } \\
\text { Janeiro, São Paulo, Paraná, } \\
\text { Santa Catarina, Rio Grande } \\
\text { do Sul }\end{array}$ \\
\hline P. planiventris (Wiedemann) & $\begin{array}{l}\text { Mexico, Guatemala, Nicaragua, } \\
\text { Costa Rica, Panama, Colombia, } \\
\text { Venezuela, Trinidad, Guyana, } \\
\text { Surinam, French Guiana, Ecuador, } \\
\text { Peru, Brazil, Bolivia }\end{array}$ & $\begin{array}{l}\text { Acre, Rondônia, Amazonas, } \\
\text { Pará, Goiás, Maranhão }\end{array}$ \\
\hline P. punctiger (Enderlein) & Brazil & $\begin{array}{l}\text { Espírito Santo, Rio de Janeiro, } \\
\text { Paraná }\end{array}$ \\
\hline P. roseni (Enderlein) & Mexico, Guatemala, Panama & \\
\hline P. rothschildi (Austen) & Colombia, Ecuador, Brazil, Bolivia & Amazonas \\
\hline P. splendidus Austen & Panama & - \\
\hline P. subsignatus (Enderlein) & Panama & - \\
\hline P. tabaninus Thunberg & $\begin{array}{l}\text { Lesser Antilles, Trinidad, } \\
\text { Guatemala, Panama, Colombia, } \\
\text { French Guiana, Peru, Brazil, } \\
\text { Bolivia }\end{array}$ & $\begin{array}{l}\text { Amazonas, Pará, Amapá, } \\
\text { Bahia, Minas gerais, Espírito } \\
\text { Santo, Rio de Janeiro, São } \\
\text { Paulo, Santa Catarina }\end{array}$ \\
\hline P. vittatus (Wiedemann) & $\begin{array}{l}\text { Trininad, Colombia, Venezuela, } \\
\text { Surinam, French Guiana, Peru, } \\
\text { Brazil, Bolivia }\end{array}$ & $\begin{array}{l}\text { Acre, Amazonas, Pará, Amapá, } \\
\text { Mato Grosso, Bahia, Minas } \\
\text { Gerais, Espírito Santo, São } \\
\text { Paulo }\end{array}$ \\
\hline P. zoos (Enderlein) & Mexico & - \\
\hline
\end{tabular}

location in the city of Uberaba, Minas Gerais State, previous geographical distribution of $P$. pictus is restricted to southern and southeastern Brazil, Paraguay and Argentina (Table 1; Fig. 3).

The new occurrence of $P$. pictus in the core area of the Cerrado Bioma reported here increases the geographical distribution of this fly to the central Brazilian Plateau (Fig. 3).

\section{ACKNOWLEDGMENTS}

This research was developed with grants from Conselho Nacional de Desenvolvimento Científico e Tecnológico - CNPQ (JRPL). We are also grateful to João Victor Pujol Luz for photography and revision of the manuscript. To Dr. Reginaldo Constantino and Dr. Ricardo Bonfim Machado for help using GMT and map edition. To Dr. Carlos Eduardo Guimarães Pinheiro, Dr. Martin Hauser and MSc Diego Aguilar Fachin for reading the manuscript and making helpful comments and suggestions.

\section{REFERENCES}

Amat, E.C. 2005. New records of timber flies (Diptera: Pantophthalmidae) from Colombia. Entomotropica, 20: 95-96.

Andrade, E.N. de. 1930. Subsidios para a entomologia agrícola brasileira. VIII. Pesquizas sobre a biologia da mosca da madeira, Pantophthalmus pictus (Wied., 1821). Archivos do Instituto Biológico de São Paulo, 3: 249-286.

Biavati, G.M.; Santana, F.H.A. \& Pujol-Luz, J.R. 2010. A checklist of Calliphoridae blowflies (Insecta, Diptera) associated with a pig carrion in Central Brazil. Journal of Forensic Sciences, 55: 1603-1606. D0I

Carrera, M. \& d'Andretta M.A.V. 1957. Sôbre a família Pantophtalmidae (Diptera). Arquivos de Zoologia de São Paulo, 10: 253-330.

Fachin, D.A.; Kosmann, C.; Pujol-Luz, C.V.A. \& Pujol-Luz, J.R. 2017. Lista das espécies de Pantophthalmidae e Stratiomyidae (Diptera, Stratiomyiomorpha) do estado do Mato Grosso do Sul, Brasil. Iheringia (Série Zoologia), 107(supl.): 1-5. D0I

Greene, C.T. \& Urich, F.W. 1931. The immature stages of Pantophthalmus tabaninus Thunberg, with biological notes. Transactions of Entomological Society of London, 79: 277-282.

Harterreiten-Souza, E.S.; Sujii, E.R. \& Pujol-Luz, J.R. 2014. A new species of the genus Micropeza Meigen (Diptera: Micropezidae) from Brazil. Zootaxa, 3827: 392-396. DOI

Myers, N.; Mittermeier, R.A.; Mittermeier, C.G.; Fonseca, G.A.B. \& Kent, J. 2000. Biodiversity hotspots for conservation priorities. Nature, 403: 853-858.

Papavero, N. 2009a. Catalogue of Neotropical Pantophthalmidae. Neotropical Diptera, 19: 1-11.

Papavero, N. 2009b. Manual of Neotropical Diptera. Pantophthalmidae. Neotropical Diptera, 20: 1-11.

Pujol-Luz, J.R. \& Pujol-Luz, C.V.A. 2014. Pantophthalmidae. In: Roig-Juñent S.; Claps, L.E. \& Morrone, J.J. (Eds.). Biodiversidad de Artrópodos Argentinos. San Miguel de Tucumán, Editorial INSUE-UNT. v. 4, p. 391-397.

Thorpe, W.H. 1934. Observations on the structure, biology and systematic position of Pantophthalmus tabaninus Thunb. (Diptera, Pantophthalmidae). Transactions of Entomological Society of London, 82: 5-22.

Val, F.C. 1976. Systematics and evolution of the Pantophthalmidae (Diptera, Brachycera). Arquivos de Zoologia de São Paulo, 27: 51-164.

Val, F.C. 1992. Pantophthalmidae of Central America and Panama (Diptera). In: Quintero, D. \& Aiello, A. (Eds.). Insects of Panama and Mesoamerica. Selected Studies. Oxford, Oxford University Press. cap. 40, p. 600-610.

Wessel, P. \& Smith, W.H.F. 1998. New, improved version of Generic Mapping Tools released EOS. Transactions of the American Geophysical Union, 79: 579.

Wolff, M.; Ramos-Pastrana, Y. \& Vallejo, F. 2016. Family Pantophthalmidae. Zootaxa, 4122: 306-311. D01 